\title{
A Build-in Self-Test Technique for RF Low-Noise Amplifiers
}

\author{
Yen-Chih Huang, Student Member, IEEE, Hsieh-Hung Hsieh, Student Member, IEEE, and \\ Liang-Hung Lu, Member, IEEE
}

\begin{abstract}
A built-in self-test (BIST) technique suitable for RF low-noise amplifiers (LNAs) is presented in this paper. With fully integrated amplitude detectors and logarithmic amplifiers, the BIST module can be employed as a generic platform for gain extraction of the device-under-test (DUT) without expensive testing instruments, while maintaining a reasonable hardware overhead and minimum loading effects to the DUT. Using a $0.18-\mu \mathrm{m}$ CMOS process, a 5-GHz variable-gain LNA with the proposed BIST module is implemented. Based on the experimental results, on-chip gain extraction of the LNA has been demonstrated with an error less than $1 \mathrm{~dB}$ for various gain modes. The additional chip area required for the BIST functionality measures $0.042 \mathrm{~mm}^{2}$, which is considerably small compared with the physical size of the RF amplifiers.
\end{abstract}

Index Terms-Amplitude detectors, built-in self-test (BIST), logarithmic amplifiers, low-noise amplifiers (LNAs), RF testing.

\section{INTRODUCTION}

$\mathbf{T}$ HE fast-growing market in personal communications has motivated the development of system-on-a-chip (SoC) solutions for wireless devices in a standard CMOS process. By integrating the RF frontends, baseband transceivers, and digital circuitry on the same Si substrate, the lifetime of the battery-operated electronics is prolonged due to the absence of off-chip components and power-consuming buffers. Besides, the chip area and the fabrication cost can also be reduced, leading to attractive features for mass production. However, with the increasing complexity in the circuit design, test of the fully integrated systems has become one of the most challenging tasks to take full advantage of the SoC implementations. Over the past few years, efforts have been made in the research of the built-in self-test (BIST), and the developed techniques have been extensively utilized in digital designs such that the functionality of the fabricated circuits can be verified without expensive testing instruments. Nevertheless, due to the variety of the signal forms and the difficulties in the performance extraction at higher frequencies, the BIST techniques for the RF integrated circuits are still in the early-stage development [1]-[5].

In an RF receiver frontend, the low-noise amplifier (LNA) is typically employed as the first active building block to provide sufficient amplification for the incoming signals while maintaining a minimum noise figure. Conventionally, the test of the

Manuscript received July 17, 2007; revised October 4, 2007. This work was supported in part by the National Science Council under Grant 96-2220-E-002-026 and Grant 96-2220-E-002-028.

The authors are with the Department of Electrical Engineering and Graduate Institute of Electronics Engineering, National Taiwan University, Taipei 10617,

Taiwan, R.O.C. (e-mail: lhlu@cc.ee.ntu.edu.tw).

Digital Object Identifier 10.1109/TMTT.2008.921293



Fig. 1. Conceptual illustration of the proposed BIST technique.

LNA gains is considered expensive and time consuming, as it requires a vector network analyzer with complicated calibration procedures. In order to overcome such limitations, an on-chip test approach has been proposed by adopting a testing amplifier and peak detectors [6]. Due to the presence of the additional RF amplifier, the hardware overhead is excessively large, making it less attractive for low-cost chip implementations. Alternatively, a compact RF BIST module was reported in [7]. With the on-chip switches, terminations, and power detectors, the LNA can be tested at the cost of moderate degradation in the gain and noise figure due to the parasitics from the BIST module. In this study, a BIST technique is presented for the LNA circuits at $\mathrm{RF}$ frequencies. By employing fully integrated amplitude detectors and logarithmic amplifiers, the gain of the device-under-test (DUT) can be extracted with minimum loading effects and hardware overhead. Therefore, it can be used as a generic platform to provide screening test of the fabricated chips or online performance monitoring of integrated RF frontends.

This paper is organized as follows. Section II describes the architecture and operating principles of the proposed BIST technique. The design of the BIST modules and the DUT is provided in Section III, while experimental results and discussions are presented in Sections IV and V, respectively. Finally, a conclusion is given in Section VI.

\section{Proposed BIST Technique}

A conceptual illustration of the proposed BIST technique is depicted in Fig. 1, where a variable attenuator, a comparator, and detectors are integrated with the DUT. In order to prevent undesirable performance degradation of the DUT, a shunt BIST topology is employed such that no series on-chip switches are required. The function of the detectors is to generate an output dc voltage proportional to the amplitude of the RF signals while 


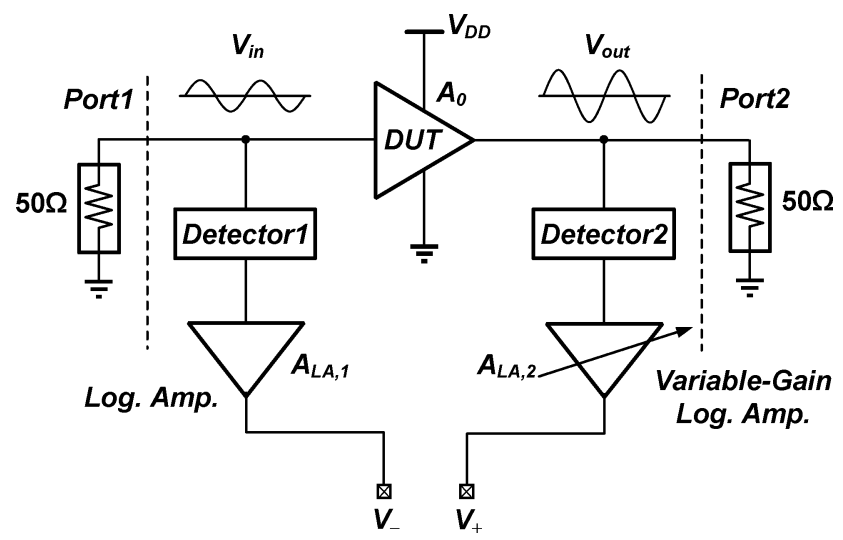

Fig. 2. RF BIST architecture for gain extraction.

the insertion gain $A_{\text {att }}$ of the variable attenuator can be adjusted by the controlled signals. Assuming that signal reflections at the ports are negligible, which is generally the case for LNA designs, the signal amplitudes at the input and output of the DUT can be, respectively, characterized by the detectors as an incident wave is applied. For a DUT with a forward gain of $A_{0}$, the dc voltages exhibiting at the inputs of the comparator become identical only if the condition $A_{0} A_{\text {att }}=1$ holds. Provided that accurate control of the variable attenuator is available, the gain of the DUT can be extracted by sweeping the insertion gain of the attenuator and specified by $A_{0}=\left(A_{\text {att }}\right)^{-1}$ when the output of the comparator changes state.

Though the RF BIST technique, as illustrated in Fig. 1, demonstrates an effective approach for gain extraction, several undesirable features undermine its feasibility in practical design cases. As the variable attenuator and DUT are operating at the same frequency, the hardware overhead becomes excessively large due to the presence of the additional RF circuit module. As a result, a significant increase in the implementation cost is expected. With the shunt topology for the testing operation, the impedance at the output port of the DUT is strongly influenced by the input characteristics of the variable attenuator, making it virtually impossible to realize a general-purpose BIST module suitable for a wide variety of RF circuits. Besides, in order to perform valid gain extraction, precise control of the attenuation is mandatory. Provided typical process variations and device mismatch in a standard CMOS technology, it is considered a challenging task for the circuit implementations, especially at multigigahertz frequencies.

Based on similar operating principles, a modified BIST architecture, as depicted in Fig. 2, is proposed to facilitate the circuit design by replacing the RF attenuator with a variable-gain logarithmic amplifier. To evaluate the forward gain, two identical amplitude detectors in the shunt configuration are employed at the input and output ports of the DUT, respectively. As the incident wave is propagating in the signal path, the detectors exhibit an output dc voltage proportional to the square of the voltage amplitude. A simplified transfer function of the amplitude detector is given by

$$
V_{o}=k_{1} V_{i}^{2} .
$$

It is noted that the only purpose for the amplitude detectors is to extract the voltage amplitude of the traveling waves. Given that the input impedance of the detectors is sufficiently high at the frequencies of interest, the loading effects to the DUT can be effectively minimized and the developed BIST modules can be generally utilized for a variety of RF integrated circuits. In addition to the amplitude detectors, two logarithmic amplifiers are employed to generate logarithmic outputs from the voltage amplitudes at the input and output port such that the gain of the DUT can be evaluated in the decibel scale. A general form of the transfer characteristics for the logarithmic amplifiers is given by

$$
V_{o}=k_{2} \ln \left(A_{\mathrm{LA}} V_{i}\right)
$$

where $k_{2}$ is a coefficient and $A_{\mathrm{LA}}$ represents the gain of the logarithmic amplifier. Based on the BIST architecture, the input voltages at the comparator can be expressed as

$$
\begin{aligned}
& V_{-}=k_{2} \ln \left(k_{1} A_{\mathrm{LA}, 1} V_{\mathrm{in}}^{2}\right) \\
& V_{+}=k_{2} \ln \left(k_{1} A_{\mathrm{LA}, 2} V_{\text {out }}^{2}\right)=k_{2} \ln \left(k_{1} A_{\mathrm{LA}, 2} A_{0}^{2} V_{\mathrm{in}}^{2}\right) .
\end{aligned}
$$

It is apparent that the input voltages of the comparator are at the same level only if the condition

$$
\sqrt{\frac{A_{\mathrm{LA}, 1}}{A_{\mathrm{LA}, 2}}}=A_{0}
$$

holds. For the circuit implementations, the input logarithmic amplifier is designed to provide a fixed gain, while a logarithmic amplifier with a variable gain is employed for the output BIST module. Again, by sweeping the value of $A_{\mathrm{LA}, 2}$ in predetermined manners, the gain of the DUT can be effectively extracted without external testing instruments.

\section{CirCUit IMPLEMENTATIONS}

With the proposed BIST technique, the amplitude detectors and logarithmic amplifiers are integrated with the DUT, which is a 5-GHz LNA in this particular circuit implementation, for on-chip gain extraction. The circuit topologies and design considerations of the individual building blocks are presented as follows.

\section{A. Amplitude Detectors}

As indicated in Section II, the amplitude detector generates a dc output voltage proportional to the square of the voltage amplitude at the observation point along the signal path. Typically, a high input impedance is desirable for the detector such that the loading effect and the influence on the performance of the DUT can be minimized. In order to serve as a general-purpose BIST module, it is required that the amplitude detector exhibits valid transfer characteristics over a wide frequency band with sufficient dynamic range. According to the performance requirements, one of the most widely used techniques for amplitude detection is to utilize the exponential $I-V$ characteristics of a bipolar transistor [8], [9]. For circuit implementations in a CMOS process, similar device properties can be realized by biasing the transistors in the subthreshold region [2], [7] and the schematic of the amplitude detector is shown in Fig. 3. 


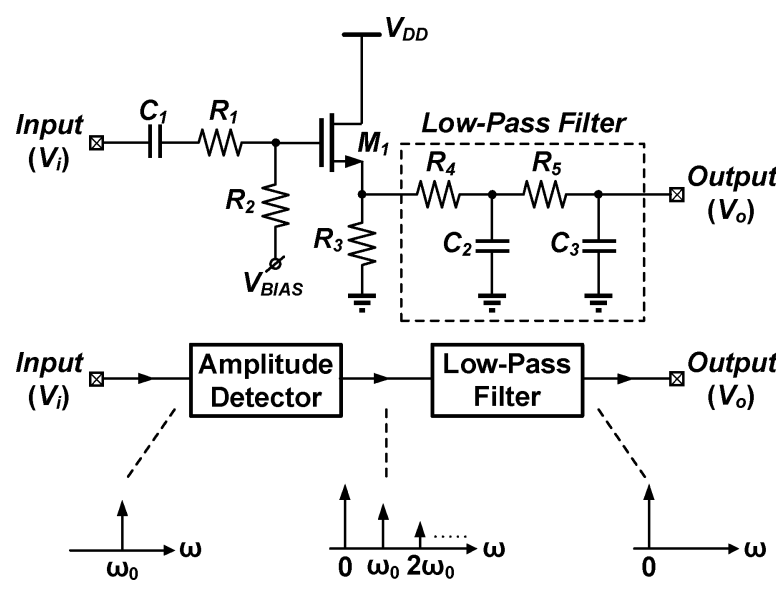

Fig. 3. Circuit schematic of the built-in amplitude detector.

At the input of the amplitude detector, the gate bias voltage of $M_{1}$ is provided through the resistor $R_{2}$, while the series capacitor $C_{1}$ is adopted for dc blocking. Considering the input impedance and the voltage division ratio, large resistance values are required for $R_{1}$ and $R_{2}$ with $R_{2} / R_{1} \gg 1$. In order to ensure the subthreshold operation of $M_{1}$, a current-limiting resistor $R_{3}$ is employed. It is noted that the $R C$ network at the output of the amplitude detector serves as a second-order low-pass filter with a cutoff frequency of $10 \mathrm{MHz}$. With a small-signal sinusoidal $V_{a} \cos \omega t$ at the gate of the MOS transistor in the subthreshold region, the saturated drain current is approximated by the power series as [10]

$$
\begin{aligned}
I_{d}= & I_{0} \exp \left(\frac{V_{\mathrm{GS}}-V_{\mathrm{th}}+V_{a} \cos \omega t}{n V_{T}}\right) \\
\approx & I_{D 0}\left[1+\frac{V_{a}}{n V_{T}} \cos \omega t+\frac{1}{2}\left(\frac{V_{a}}{n V_{T}}\right)^{2} \cos ^{2} \omega t\right] \\
= & I_{D 0}\left[1+\left(\frac{V_{a}}{2 n V_{T}}\right)^{2}\right]+I_{D 0}\left(\frac{V_{a}}{n V_{T}}\right) \cos \omega t \\
& +I_{D 0}\left(\frac{V_{a}}{2 n V_{T}}\right)^{2} \cos 2 \omega t
\end{aligned}
$$

where $V_{T}$ is the thermal voltage, $V_{\mathrm{th}}$ is the threshold voltage, $n$ is the subthreshold slop factor, $I_{0}$ is a process-dependent current, and $I_{D 0}$ represents the dc-bias current of the transistor. With proper design parameters for the low-pass filter to reject the fundamental and the harmonic components, the output voltage of the amplitude detector is simply a dc voltage as

$$
V_{o}=I_{D 0} R_{3}+I_{D 0} R_{3}\left(\frac{V_{a}}{2 n V_{T}}\right)^{2} .
$$

Note that, by employing a replica circuit to cancel out the first term in (7), a transfer function of the amplitude detector in the form of (1) is thus achieved.

The proposed amplitude detector is implemented in a $0.18-\mu \mathrm{m}$ CMOS process, and the circuit parameters are tabulated in Table I. Note that the performance of the detector is strongly influenced by the nMOS transistor. In this particular design, the aspect ratio and gate bias are chosen such that the transistor operates in the subthreshold region while maintaining
TABLE I

DESIGNEd PARAMETERS OF THE AMPLITUdE DETECTOR

\begin{tabular}{|c|c|}
\hline Devices & Designed Parameters \\
\hline$M_{1}$ & $10 \mu \mathrm{m} / 0.18 \mu \mathrm{m}$ \\
\hline$C_{l}$ & $1 \mathrm{pF}$ \\
\hline$C_{2}, C_{3}$ & $5 \mathrm{pF}$ \\
\hline$R_{1}$ & $78 \Omega$ \\
\hline$R_{2}$ & $5 \mathrm{k} \Omega$ \\
\hline$R_{3}$ & $50 \mathrm{k} \Omega$ \\
\hline$R_{4}, R_{5}$ & $28 \mathrm{k} \Omega$ \\
\hline
\end{tabular}

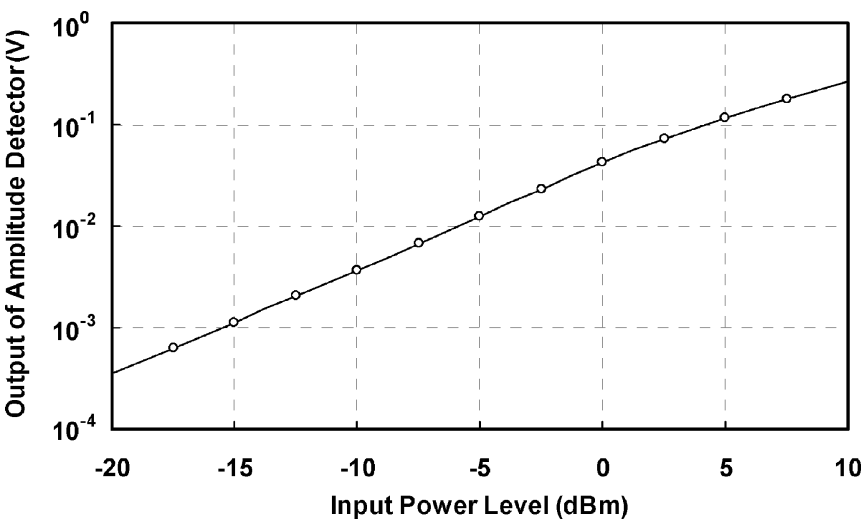

Fig. 4. Simulated output voltage of the amplitude detector as a function of the input power level with a $50-\Omega$ load.

minimum capacitive loading at the input. Fig. 4 shows the simulated output voltage versus the input power level. From the plot in the logarithmic scale, the detector exhibits excellent linearity in the transfer characteristics with an input power ranging from -20 to $10 \mathrm{dBm}$. As the input power level further increases, the higher order terms in the drain current become significant such that the output voltage deviates from the derivation in (7). Therefore, the dynamic range for this particular BIST design is approximately $30 \mathrm{~dB}$, which is sufficient for gain extraction in typical LNA circuits at multigigahertz frequencies.

\section{B. Logarithmic Amplifiers}

In order to facilitate the gain extraction in decibels, logarithmic amplifiers are employed to compare the signal amplitudes at the input and output port of the DUT. Fig. 5(a) shows the circuit schematic of the op-amp-based logarithmic amplifier with a constant gain. Provided a dc voltage at the input, the output of the logarithmic amplifier can be expressed as

$$
V_{o}=V_{\text {ref }}-n_{D} V_{T} \ln \left(\frac{V_{i}-V_{\text {ref }}}{I_{s} R_{\mathrm{LA} 1}}\right)
$$

where $I_{s}$ and $n_{D}$ represent the reverse saturation current and the ideality factor of the diode, respectively. Based on the proposed BIST architecture, as specified in Fig. 2, a logarithmic amplifier with a variable gain is required at the output of the DUT. By comparing (2) and (8), it is obvious that the gain of the circuit in Fig. 5(a) can be effectively manipulated by the value of the input resistor. The schematic of the variable-gain logarithmic amplifier is shown in Fig. 5(b), where a resistor array with 4-bit 


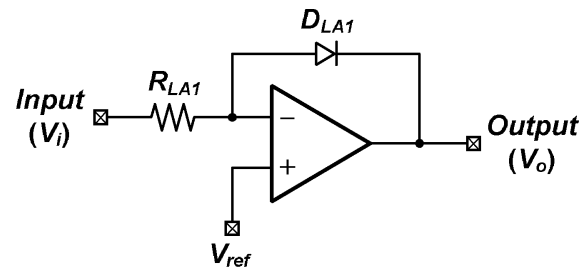

(a)

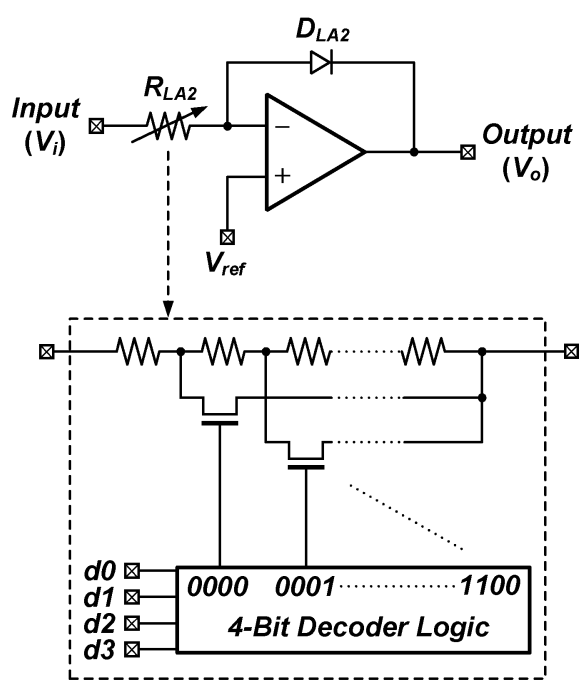

(b)

Fig. 5. Circuit implementations of the logarithmic amplifiers with: (a) constant gain and (b) variable gain.

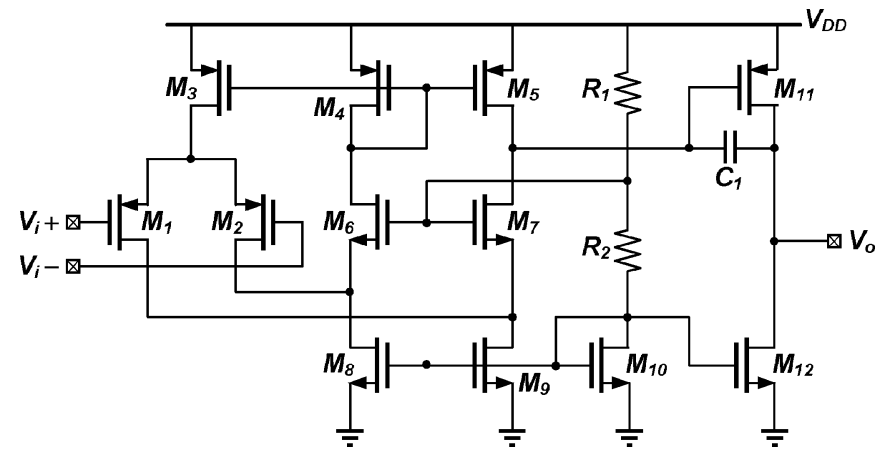

Fig. 6. Circuit schematic of the op-amp used in the logarithmic amplifier.

controlled signals and a decoder logic is employed in this particular design for the gain adjustment.

The design of the logarithmic amplifiers starts with the op-amp circuit. With a two-stage folded architecture, as indicated in Fig. 6, the design parameters are tabulated in Table II. Based on circuit simulations, the op-amp exhibits an open-loop gain of $70 \mathrm{~dB}$ and a phase margin of $80^{\circ}$. As for the diodes, lateral bipolar devices in a standard CMOS technology are adopted. Fig. 7 shows the simulated transfer characteristics of the logarithmic amplifier with various gain factors. It is noted that the circuit parameters for the input and output logarithmic amplifiers are identical and resistors with matched device layout are employed such that the error in the BIST operations can be minimized.
TABLE II

DESIGNED PARAMETERS OF THE OP-AMP USED IN THE LOGARITHMIC AMPLIFIER

\begin{tabular}{|c|c|}
\hline Devices & Designed Parameters \\
\hline$M_{1}, M_{2}$ & $4 \mu \mathrm{m} / 2 \mu \mathrm{m}$ \\
\hline$M_{3}$ & $8 \mu \mathrm{m} / 1 \mu \mathrm{m}$ \\
\hline$M_{4}, M_{5}$ & $4 \mu \mathrm{m} / 1 \mu \mathrm{m}$ \\
\hline$M_{6}-M_{9}$ & $1 \mu \mathrm{m} / 1 \mu \mathrm{m}$ \\
\hline$M_{10}$ & $10 \mu \mathrm{m} / 1 \mu \mathrm{m}$ \\
\hline$M_{11}$ & $400 \mu \mathrm{m} / 0.25 \mu \mathrm{m}$ \\
\hline$M_{12}$ & $50 \mu \mathrm{m} / 0.25 \mu \mathrm{m}$ \\
\hline$R_{1}$ & $17 \mathrm{k} \Omega$ \\
\hline$R_{2}$ & $8.6 \mathrm{k} \Omega$ \\
\hline$C_{1}$ & $0.7 \mathrm{pF}$ \\
\hline
\end{tabular}

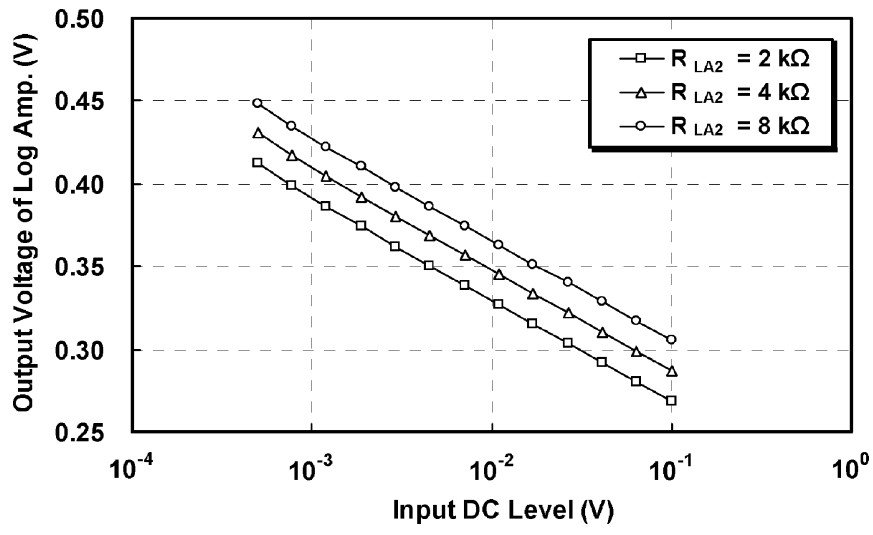

Fig. 7. Simulated output voltage of the logarithmic amplifier versus the input voltage for various values of $R_{\mathrm{LA} 2}$.

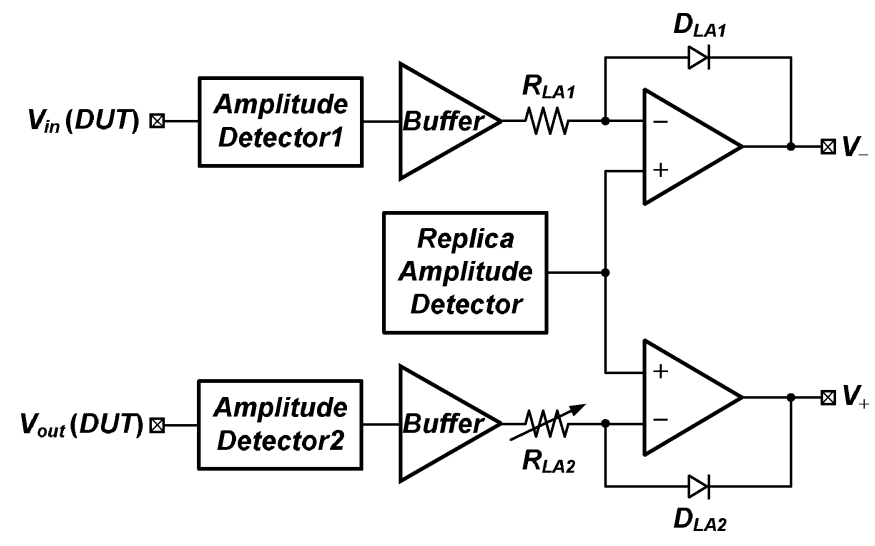

Fig. 8. Complete schematic of the proposed BIST module.

\section{Integrated BIST Module}

Fig. 8 shows the fully integrated BIST module, which is composed of two amplitude detectors, two unit-gain buffers, two logarithmic amplifiers, and a replica circuit. In the proposed architecture, the replica circuit, which is identical to the amplitude detectors, generates the input-independent term $I_{D 0} R_{3}$ as the reference voltage $V_{\text {ref }}$ for the logarithmic amplifiers. Base on 
TABLE III

RESISTOR RATIOS AND GAIN LEVELS FOR THE BIST DESIGN

\begin{tabular}{|c|c|c|}
\hline 4-Bit Code & $R_{L A 2} / R_{L A 1}$ & Gain $(\mathrm{dB})$ \\
\hline 0000 & 1 & 0 \\
\hline 0001 & 2 & 3.0 \\
\hline 0010 & 4 & 6.0 \\
\hline 0011 & 8 & 9.0 \\
\hline 0100 & 10 & 10.0 \\
\hline 0101 & 13 & 11.1 \\
\hline 0110 & 16 & 12.0 \\
\hline 0111 & 20 & 13.0 \\
\hline 1000 & 26 & 14.1 \\
\hline 1001 & 32 & 15.1 \\
\hline 1010 & 40 & 16.0 \\
\hline 1011 & 50 & 17.0 \\
\hline 1100 & 63 & 18.0 \\
\hline
\end{tabular}



Fig. 9. Schematic of the LNA used as the DUT in this design.

(7) and (8), the output voltages of the BIST module are given by

$$
\begin{aligned}
& V_{-}=I_{D 0} R_{3}-2 n_{D} V_{T} \ln \left(\frac{V_{i}}{2 n V_{T}}\right)-n_{D} V_{T} \ln \left(\frac{I_{D 0} R_{3}}{I_{s} R_{\mathrm{LA} 1}}\right) \\
& V_{+}=I_{D 0} R_{3}-2 n_{D} V_{T} \ln \left(\frac{A_{0} V_{i}}{2 n V_{T}}\right)-n_{D} V_{T} \ln \left(\frac{I_{D 0} R_{3}}{I_{s} R_{\mathrm{LA} 2}}\right) .
\end{aligned}
$$

Therefore, as the condition for $V_{+}=V_{-}$holds, the gain expression, as indicated in (5), can be rewritten as

$$
A_{0}=10 \times \log \left(\frac{R_{\mathrm{LA} 2}}{R_{\mathrm{LA} 1}}\right)(\mathrm{dB}) .
$$

In this BIST design, a resistor array with 4-bit controlled signals and a decoder is adopted for $R_{\mathrm{LA} 2}$ and the discrete levels provided for gain extraction is shown in Table III. It is obvious that the proposed architecture can be easily modified to achieve the desirable range and resolution by rearranging the resistor array. As indicated in (11), the accuracy of the gain extraction is solely depending on the ratio of the resistors $R_{\mathrm{LA} 1}$ and $R_{\mathrm{LA} 2}$. Provided the device matching properties in standard CMOS technologies, the proposed BIST technique is well suited for general-purpose RF applications with sufficient accuracy.
TABLE IV

DESIGNED PARAMETERS OF THE LNA

\begin{tabular}{|c|c|}
\hline Devices & Designed Parameters \\
\hline$M_{1}, M_{3}$ & $250 \mu \mathrm{m} / 0.18 \mu \mathrm{m}$ \\
\hline$M_{2}$ & $300 \mu \mathrm{m} / 0.18 \mu \mathrm{m}$ \\
\hline$L_{1}$ & $3.3 \mathrm{nH}$ \\
\hline$L_{2}, L_{4}$ & $0.9 \mathrm{nH}$ \\
\hline$L_{3}$ & $2.2 \mathrm{nH}$ \\
\hline$L_{5}$ & $1.2 \mathrm{nH}$ \\
\hline$C_{1}$ & $0.85 \mathrm{pF}$ \\
\hline$C_{2}$ & $1.0 \mathrm{pF}$ \\
\hline
\end{tabular}

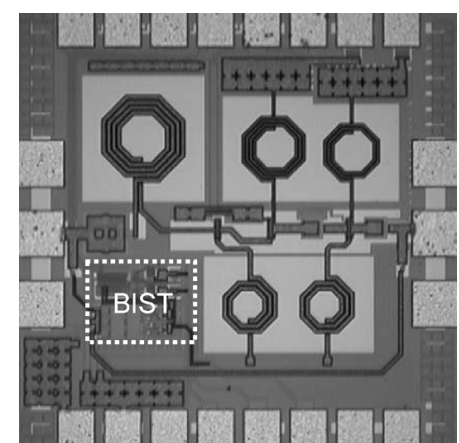

Fig. 10. Microphotograph of the fabricated circuit.

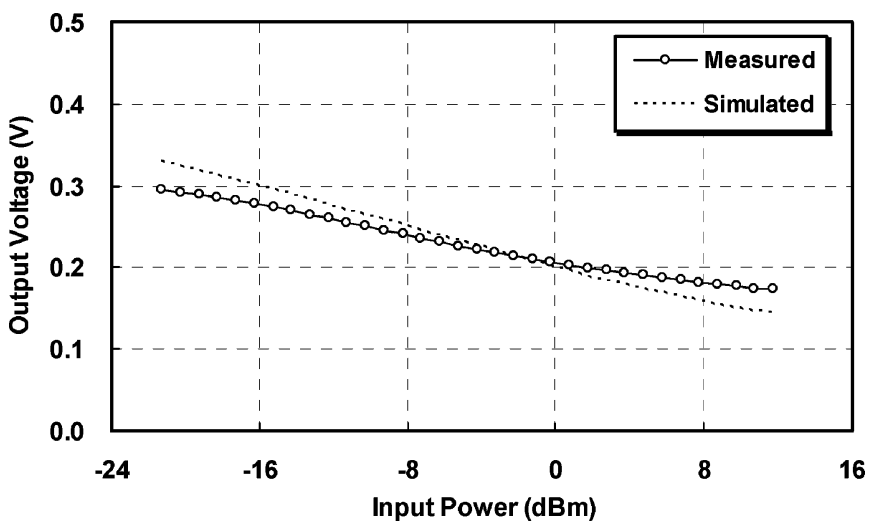

Fig. 11. Measured transfer characteristics of the BIST module including the amplitude detector and logarithmic amplifier.

TABLE V

Performance Summary OF THE BIST Module

\begin{tabular}{|c|c|}
\hline \multicolumn{2}{|c|}{ Performance of BIST Modules } \\
\hline Technology & $0.18-\mu \mathrm{m} \mathrm{CMOS}$ \\
\hline Area & $0.042 \mathrm{~mm}^{2}$ \\
\hline Supply Voltage & $1.8 \mathrm{~V}$ \\
\hline DC Power & $7.2 \mathrm{~mW}$ \\
\hline Operating Frequency & $1 \sim 10 \mathrm{GHz}$ \\
\hline Dynamic Range & $30 \mathrm{~dB}$ \\
\hline
\end{tabular}

\section{DUT}

To verify the functionality of the proposed BIST modules, a 5-GHz variable-gain LNA is employed as the DUT for demonstration. Fig. 9 shows the circuit schematic of the LNA where 


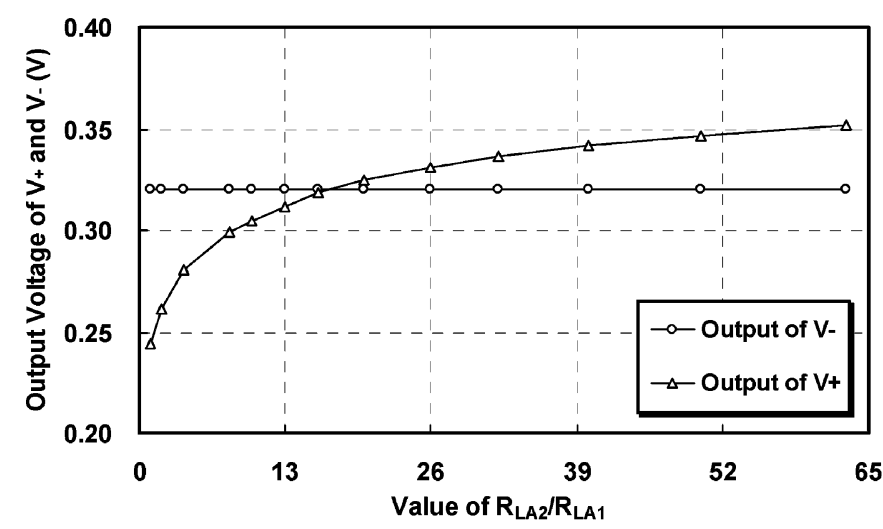

(a)

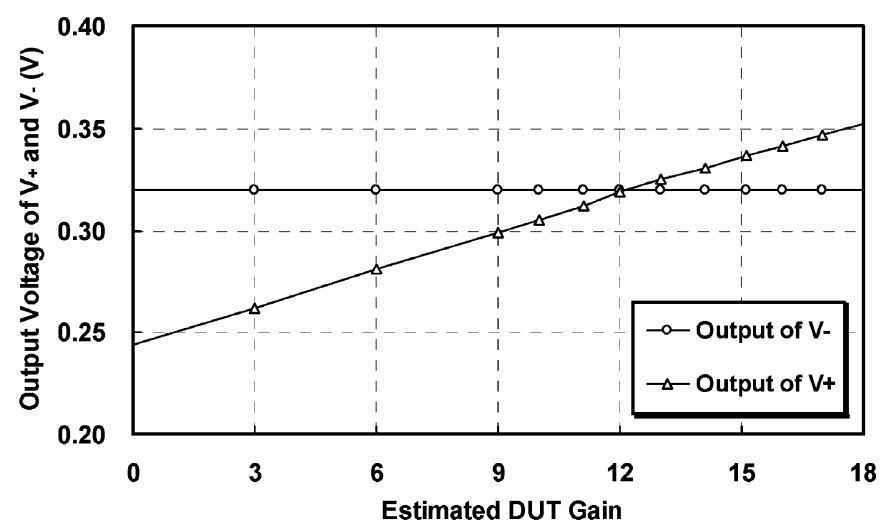

(b)

Fig. 12. Measured BIST outputs $V_{+}$and $V_{-}$versus: (a) resistor ratio $\left(R_{\mathrm{LA} 2} / R_{\mathrm{LA} 1}\right)$ and (b) estimated gain of DUT at the high-gain mode.

$M_{1}$ and $M_{2}$ represent the first cascode gain stage, and $M_{3}$ is the second gain stage with a common-source configuration. The inductors $L_{1}$ and $L_{2}$ are employed as the input matching network, while the output matching is achieved by $L_{5}$ and $C_{2}$. In addition, the $L C$ network of $L_{3}$ and $C_{1}$ is adopted for inter-stage matching in consideration of the amplifier gain and the circuit stability. In this LNA design, the variable gain is achieved by adjusting the bias voltage $V_{G 1}$. As $V_{G 1}$ varies, the LNA circuit can operate at various gain modes while maintaining the desirable impedance matching at the input and output port. The circuit parameters of the DUT are summarized in Table IV.

\section{EXPERIMENTAL RESULTS}

Using a $0.18-\mu \mathrm{m}$ CMOS process, the $5-\mathrm{GHz}$ variable-gain LNA and the proposed BIST module are integrated in a single chip. Fig. 10 shows a die photograph of the fabricated circuit. In this particular design, the total chip area excluding the pads is $0.75 \times 0.74 \mathrm{~mm}^{2}$, while the real estate accounted for the BIST functionality is approximately $7.6 \%$ of the overall active area. Operated at a supply voltage of $1.8 \mathrm{~V}$, the current consumptions for the DUT and BIST module are 8 and $4 \mathrm{~mA}$, respectively. The performance of the fabricated circuits was characterized by on-wafer probing.

Before performing gain extraction of the DUT, the transfer characteristics of the building blocks were evaluated. By sweeping the power level of the 5-GHz sinusoidal input provided by an external source, the output voltage of the cascaded stages including the amplitude detector and logarithmic amplifier is shown in Fig. 11, exhibiting good agreement with the results from circuit simulation. For accurate gain extraction, the required dynamic range of the BIST module is specified by the DUT properties as

$$
\mathrm{DR} \geq\left(P_{\mathrm{in}-1 \mathrm{~dB}}+A_{0}-1\right)-P_{\mathrm{in}-1 \mathrm{~dB}}=A_{0}-1
$$

where $P_{\mathrm{in}-1 \mathrm{~dB}}$ is the input $1-\mathrm{dB}$ compression point of the DUT. Typically, sufficient margin is recommended to have a robust design against the process variations. Based on the measurement results, the BIST module demonstrates a dynamic range of $30 \mathrm{~dB}$. Table V summaries the circuit performance of the BIST modules.

With a 5-GHz sinusoidal input at a power level of $-20 \mathrm{dBm}$, the gain extraction was performed on the DUT at the high gain mode by observing the output voltages of the BIST module. As the controlled bits vary from 0000 to 1100 , the equivalent resistance for $R_{\mathrm{LA} 2}$ increases accordingly. Fig. 12(a) illustrates the measured BIST outputs $V_{+}$and $V_{-}$versus the resistance ratio $R_{\mathrm{LA} 2} / R_{\mathrm{LA} 1}$. According to the experimental results, the crossing point of the output voltages $V_{+}$and $V_{-}$takes place with a value of $R_{\mathrm{LA} 2} / R_{\mathrm{LA} 1}$ between 16-20. Therefore, it can be concluded from (11) that the gain of the DUT falls within the interval from 12.0 to $13.0 \mathrm{~dB}$. Alternatively, the BIST output voltage can be plotted as a function of the estimated DUT gain in decibels, and the results are shown in Fig. 12(b). Theoretically, the trace of $V_{+}$in Fig. 12(b) is a straight line. As a result, the DUT gain can be estimated by interpolating the data points, leading to an extracted value of $12.4 \mathrm{~dB}$. In order to verify the functionality of the BIST technique, the DUT was tested using a network analyzer as the benchmark. Compared with the measured gain of $12.9 \mathrm{~dB}$, the BIST technique provides gain extraction with sufficient accuracy. As for the gain extraction of the DUT operating at the low-gain mode, the similar BIST procedures were carried out and the experimental results are shown in Fig. 13, indicating an extracted gain of $9.5 \mathrm{~dB}$ with respect to a measured value of $9.3 \mathrm{~dB}$. The same gain extraction technique can also be applied to evaluate the in-band frequency response of the DUT provided that the low reflection coefficients are maintained at the input and the output port. By performing the BIST procedures at various input frequencies, the extracted frequency responses of the DUT are illustrated in Fig. 14, showing good agreement with the measured values in the vicinity of the center frequency.

\section{Discussions}

\section{A. Accuracy for Gain Extraction}

One of the most important features in the proposed BIST technique is that the gain extraction of the DUT depends on the resistance ratios instead of the transfer characteristics of the build-in circuit modules. Besides, the on-chip terminations and switches are no longer required. Rather complicated calibration procedures, which are employed in [1] and [7], can be prevented. As far as the circuit implementation is concerned, the accuracy is solely limited by device mismatch. In this particular design, the input and output BIST modules are implemented with a matched layout in close proximity to minimize the errors. In addition, strategies such as multifinger transistors, dummy cells, 


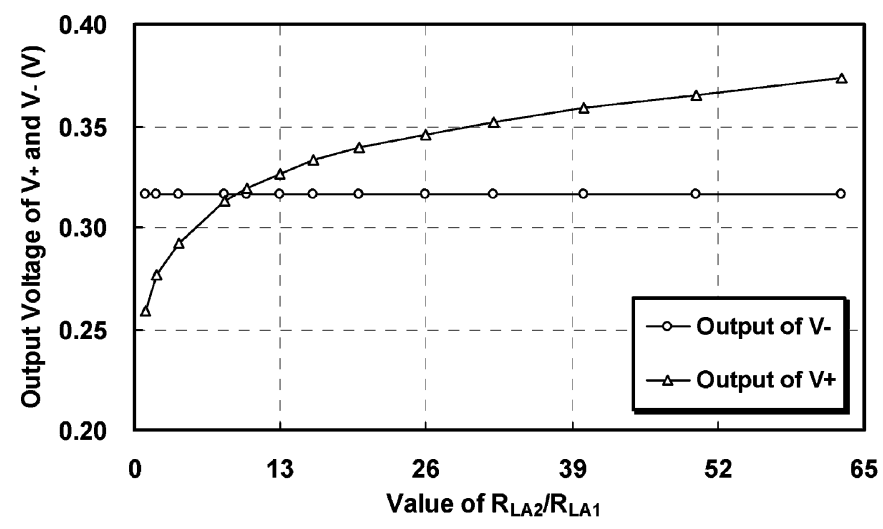

(a)

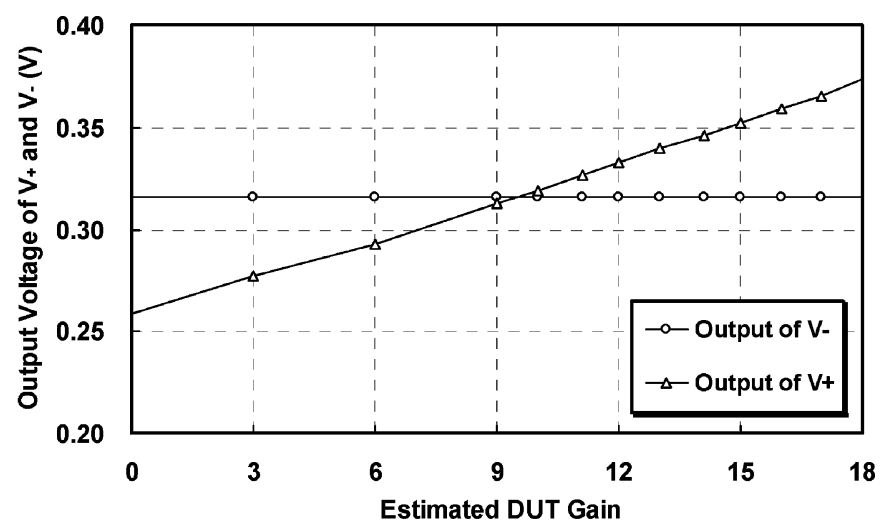

(b)

Fig. 13. Measured BIST outputs $V_{+}$and $V_{-}$versus: (a) resistor ratio $\left(R_{\mathrm{LA} 2} / R_{\mathrm{LA} 1}\right)$ and (b) estimated gain of the DUT at the low-gain mode.



(a)

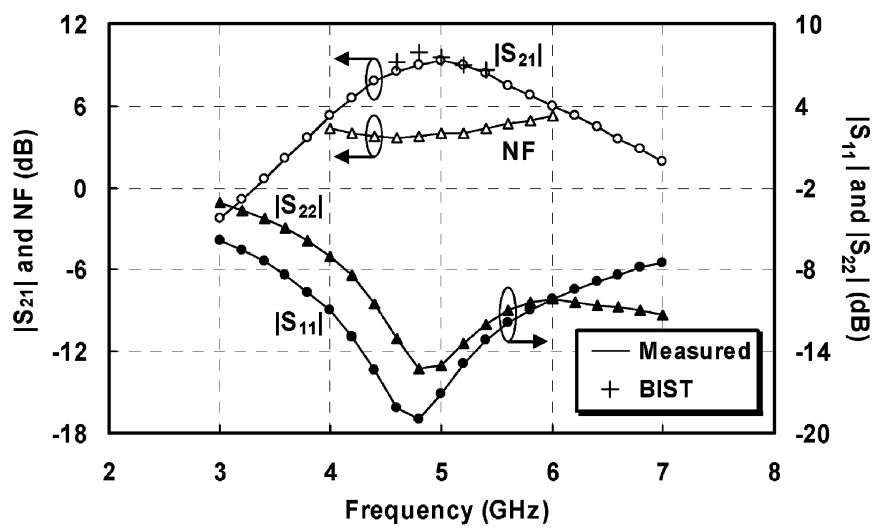

(b)

Fig. 14. Measured and the BIST extracted frequency responses of the DUT operating at: (a) high-gain and (b) low-gain modes.

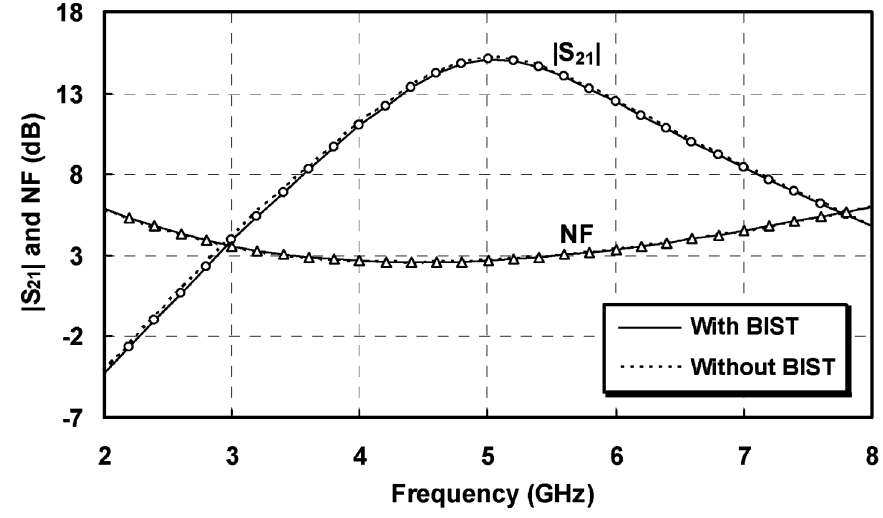

(a)

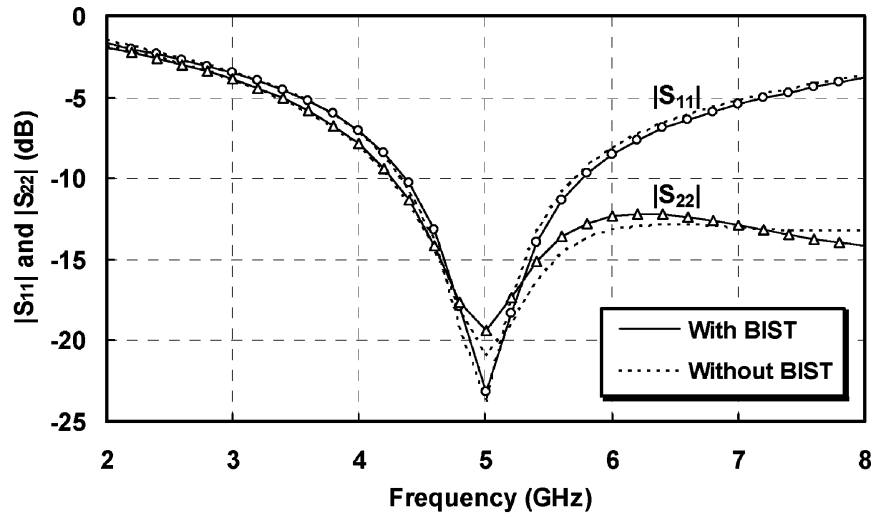

(b)

Fig. 15. Simulated DUT performance with and without the BIST modules at the high-gain mode. (a) Gain $\left|S_{21}\right|$ and noise figure. (b) Reflection coefficients $\left|S_{11}\right|$ and $\left|S_{22}\right|$.

and common-centroid symmetry [11] are also adopted. With typical process variations in the CMOS technologies, the proposed circuits can be utilized to perform gain extraction with sufficient accuracy.

It is noted that the operating principle of the BIST technique is based on a DUT with input and output matched to the port impedance such that the signal power can be evaluated by its voltage amplitude. Therefore, detection errors are introduced in the test if the DUT is not perfectly matched. From (9) and
(10), the gain error resulted from the impedance mismatch in the proposed technique is estimated by

$$
\varepsilon=20 \times \log \left|1+\Gamma_{\text {in }}\right|-20 \times \log \left|1+\Gamma_{\text {out }}\right|(d B)
$$

where $\Gamma_{\text {in }}$ and $\Gamma_{\text {out }}$ represent the reflection coefficients at the input and output ports, respectively. According to (13) and results from circuit simulations, an input return loss better than 
$10 \mathrm{~dB}$ is generally required for the DUT to ensure the testing accuracy in this particular BIST design.

\section{B. Influence of BIST Modules}

For conventional BIST techniques, the influence of the additional circuit modules on the DUT performance is an important issue and has to be carefully examined. In order to minimize the undesirable loading effects, amplitude detectors with high input impedance are employed in the proposed design. Besides, due to the absence of the series on-chip switches, the DUT performance degradation in terms of the gain and noise figure is thus prevented. In this study, since the circuit without the BIST modules is not fabricated, simulations are used to evaluate the influence of the additional circuitry on the performance. In Fig. 15, the simulated results of the DUT with and without the BIST modules at the high-gain mode are shown. It is clear that only unnoticeable deviations are observed between these two cases at the frequencies of interest.

\section{Applications in a Fully Integrated Frontend}

In this study, a standalone LNA is employed as the DUT for demonstration. By incorporating the BIST modules at the input and output nodes in the signal path of interest, the gain or the attenuation of the fully integrated system can also be extracted based on the similar procedures. It is noted that an incident RF signal is generally required in the BIST operation. For the test of an integrated receiver, the input stimulus can be provided either by an external source or by the transmitter output signals with a loop-back test strategy [12], [13]. As for the transmitting path, the signals generated by the baseband circuitry can be used for the test of the transmitter frontend. Therefore, the proposed BIST mechanism is well suitable for gain extraction of the RF frontends in a SoC chip.

\section{CONCLUSION}

This paper has presented the architecture and experimental results of a novel BIST technique for RF applications. With the fully integrated amplitude detectors and logarithmic amplifiers, the gain of the DUT can be extracted without expensive test instruments and a complicated calibration procedure. Using a $0.18-\mu \mathrm{m}$ CMOS process, the proposed technique has been successfully demonstrated on a 5-GHz LNA with an acceptable hardware overhead.

\section{ACKNOWLEDGMENT}

The authors would like to thank the National Chip Implementation Center (CIC), Hsinchu, Taiwan, R.O.C., for chip fabrication and the National Nano Device Laboratories (NDL), Hsinchu, Taiwan, R.O.C., for chip measurement.

\section{REFERENCES}

[1] Y.-C. Huang, H.-H. Hsieh, and L.-H. Lu, "A low-noise amplifier with integrated current and power sensors for RF BIST applications," in IEEE VLSI Test Symp., May 2007, pp. 401-406.

[2] Q. Wang and M. Soma, "RF front-end system gain and linearity built-in test," in IEEE VLSI Test Symp., Apr. 2006, pp. 228-233.

[3] T. Das et al., "Self-calibration of input-match in RF front-end circuitry," IEEE Trans. Circuits Syst. II, Exp. Briefs, vol. 52, no. 12, pp. 821-825, Dec. 2005.
[4] A. Gopalan et al., "Use of source degeneration for non-intrusive BIST of RF front-end circuits," in IEEE Int. Circuits Syst. Symp., May 2005, vol. 5, pp. 4385-4388.

[5] A. Soldo et al., "A current sensor for on-chip, non-intrusive testing of RF systems," in IEEE Int. VLSI Design Conf., Jan. 2004, pp. 1023-1026.

[6] J.-Y. Ryu, B. C. Kim, and I. Sylla, "A new low-cost RF built-in self-test measurement for system-on-chip transceivers," IEEE Trans. Instrum. Meas., vol. 55, no. 4, pp. 381-388, Apr. 2006.

[7] H.-H. Hsieh and L.-H. Lu, "Integrated CMOS power sensors for RF BIST applications," in IEEE VLSI Test Symp., Apr. 2006, pp. 229-233.

[8] T. Zhang, W. R. Eisenstadt, and R. M. Fox, "A novel 5 GHz RF power detector," in IEEE Int. Circuits Syst. Symp., May 2004, pp. 897-900.

[9] S. Kulhalli, S. Seth, and S.-T. Fu, "An integrated linear RF power detector," in IEEE Int. Circuits Syst. Symp., May 2004, pp. 625-628.

[10] B. H. Calhoun, A. Wang, and A. Chandrakasan, "Modeling and sizing for minimum energy operation in subthreshold circuits," IEEE J. SolidState Circuits, vol. 40, no. 9, pp. 1778-1786, Sep. 2005.

[11] B. Razavi, Design of Analog CMOS Integrated Circuits. New York: McGraw-Hill, 2001.

[12] M. Jarwala, L. Duy, and M. S. Heutmaker, "End-to-end test strategy for wireless systems," in IEEE Int. Test Conf., 1995, pp. 940-946.

[13] D. Lupea, U. Pursche, and H.-J. Jentschel, "RF-BIST: Loopback spectral signature analysis," in IEEE Design, Automat., Test in Eur. Conf. and Exhibition, 2003, pp. 478-483.

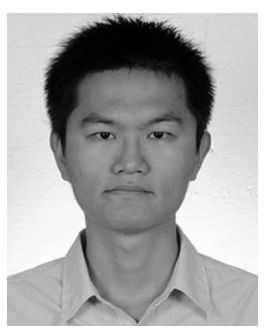

Yen-Chih Huang (S'07) was born in Taichung, Taiwan, R.O.C., in 1982. He received the B.S. degree in electrical engineering and M.S. degree in electronics engineering from National Taiwan University, Taipei, Taiwan, R.O.C., in 2005 and 2007, respectively.

His research interests include RF integrated circuits and RF testing.

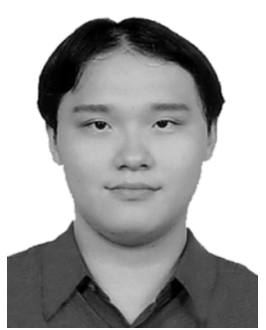

Hsieh-Hung Hsieh (S'05) was born in Taipei, Taiwan, R.O.C., in 1981. He received the B.S. degree in electrical engineering from National Taiwan University, Taipei, Taiwan, R.O.C., in 2004, and is currently working toward the Ph.D. degree in electronic engineering at National Taiwan University.

His research interests include the development of low-voltage and low-power RF integrated circuits, multiband wireless systems, RF testing, and monolithic microwave integrated circuit (MMIC) designs.

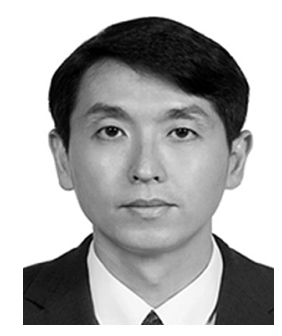

Liang-Hung Lu (M'02) was born in Taipei, Taiwan, R.O.C., in 1968. He received the B.S. and M.S. degrees in electronics engineering from National Chiao-Tung University, Hsinchu, Taiwan, R.O.C., in 1991 and 1993, respectively, and the Ph.D. degree in electrical engineering from The University of Michigan at Ann Arbor, in 2001.

During his graduate study, he was involved in SiGe HBT technology and MMIC designs. From 2001 to 2002, he was with IBM, where he was involved with low-power and RF integrated circuits for silicon-oninsulator (SOI) technology. In August 2002, he joined the faculty of the Graduate Institute of Electronics Engineering and the Department of Electrical Engineering, National Taiwan University, Taipei, Taiwan, R.O.C., where he is currently an Associate Professor. His research interests include CMOS/BiCMOS $\mathrm{RF}$ and mixed-signal integrated-circuit designs. 